\title{
Sphenoid Sinus Fat Packing in Transsphenoidal Surgery: Long-Term Fate Assessment Using Magnetic Resonance Imaging
}

\author{
Chi Sang Hwang, $\mathrm{MD}^{1}$, Kum Whang, $\mathrm{MD}, \mathrm{PhD}^{2}$, Hee Sung Chae, $\mathrm{MD}^{1}$, Hyun Su Lee, $\mathrm{MD}^{3}$, \\ Chung Seok Lee, $\mathrm{MD}^{3}$, Young Joon Seo, $\mathrm{MD}, \mathrm{PhD}^{1}$, and Dong-Joon Park, $\mathrm{MD}, \mathrm{PhD}^{1}$ \\ ${ }^{1}$ Department of Otorhinolaryngology and ${ }^{2}$ Neurosurgery, Yonsei University Wonju College of Medicine, Wonju; and \\ ${ }^{3}$ Yonsei University Wonju College of Medicine, Wonju, Korea
}

\begin{abstract}
Background and Objectives: Following the transsphenoidal approach (TSA), appropriate sphenoid sinus fat packing has been preferred to prevent postoperative cerebrospinal fluid leakage; however, studies on the behavior of fat tissue transplanted in the sphenoid sinus are lacking. This study aimed to determine the long-term fate of these fat grafts using magnetic resonance imaging (MRI).

Subjects and Method: A total of 139 postoperative MRI scans of 41 patients who underwent sphenoid sinus fat packing using the standard TSA were evaluated. Additionally, MRI time series indicating the vital fat volumes were assessed postoperatively. Results: In $82.9 \%$ of cases, the fat volumes measured in the final MRI scans declined to $<20 \%$ of the initial volumes; only $4.9 \%$ of cases exhibited declines to $>60 \%$ of the initial volume. The fat tissue volume decreased significantly with time, with a median half-life of 18 months. Typically, the sphenoid sinus was eventually almost filled with air rather than transplanted fat. In the subgroup analysis, the fat clearance rate was significantly lower in patients with residual tumors than in those without such remnants $(\mathrm{p}=0.013)$.

Conclusion: Long-term MRI surveillance of fat grafts in the sphenoid sinus revealed that the transplanted fat graft had degraded and was gradually eliminated.
\end{abstract}

KEY WORDS: Fat grafting · Graft survival · Transsphenoidal approach · Pituitary adenomas · Magnetic resonance imaging.

\section{INTRODUCTION}

Pituitary adenomas are among the most common primary intra-cranial neoplasms, with an overall estimated prevalence of approximately $16.7 \%$ in the general population. ${ }^{12)}$ When surgery is indicated, the trans-sphenoidal approach (TSA) has been the preferred access route to parasellar lesions. ${ }^{3)}$ Autologous fat transplants, vascularized mucosal flaps, or various synthetic materials can be used to support the sellar floor in cases with intra-operative risk factors for postoperative cerebrospinal fluid (CSF) fistula. ${ }^{45)}$ The merits of various techniques and materials are still debated, with selection largely dependent on the personal preferences of surgeons. ${ }^{6-8)}$ Sphenoid sinus packing using autologous abdominal fat tissues with or without lumbar drainage is still recommended for greater confidence in surgical outcomes. ${ }^{9)}$

Autologous fat grafting is becoming an increasingly popular procedure as an ideal means of soft-tissue filling that is used for facial rejuvenation or body contouring, since fat grafts are biocompatible, nonallergenic, nontoxic, easy to obtain, and synergistic with natural skin. ${ }^{10) 11)}$ Because the actual mechanism underlying fat graft survival is not fully understood, adipose cell viability and volume changes after fat grafting have become a great concern to surgeons. Although consistent scientific evidence of the long-term viability of the transferred fat is not available, the fat resorp-

Received: July 30, 2019 / Revised: September 3, 2019 / Accepted: October 9, 2019

Address for correspondence: Dong-Joon Park, MD, PhD, Department of Otorhinolaryngology, Yonsei University Wonju College of Medicine, 20 Ilsan-ro, Wonju 26426, Korea

Tel: +82-33-741-0641, Fax: +82-33-732-8287 E-mail: rhico@yonsei.ac.kr 
tion rate is reported to range from 20 to $90 \%{ }^{12)}$

The established rationale on fat graft survival is that the transplanted fat should be in contact with living tissue. ${ }^{13)}$ The TSA technique includes removal of the sphenoid sinus mucosa such that the fat grafts could survive via contact with the recipient tissue bed with potential re-establishment of the blood supply to the graft. Therefore, it is expected that in sphenoid sinus fat packing, the graft would survive for some time period. However, no studies have examined the behavior of fat tissue transplanted in the sphenoid sinus. We therefore aimed to assess the fate of such fat transplants without revision operations, using a non-invasive analysis of the MRI scan series following fat grafting using the TSA. Furthermore, the results from our study may aid in improving the overall viability of fat grafts and the predictability of the outcome of fat grafting, which is likely to be widely used in various surgeries in future.

\section{SUBJECTS AND METHODS}

\section{Ethical considerations}

This study was approved by the institutional review board (IRB) of Yonsei University Wonju College of Medicine (IRB No. 2018-05-0223). All patients provided written consent prior to surgery, and all data were retrospectively analyzed. All procedures were conducted in accordance with the ethical standards of the institutional and/or national research committee and with the 1964 Helsinki declaration and its later amendments, or with comparable ethical standards.

\section{Subject profiles}

The decisive target parameter of this study was fat volumes assessed by MRI. Inclusion was therefore restricted to the patients for whom postoperative MRI scans were available. Between January 2010 and December 2016, 41 patients diagnosed with pituitary adenoma and who had undergone surgery using the microscope-guided transseptal TSA were enrolled. All patients were aged $>19$ years and had undergone postoperative MRI scans for a minimum follow-up period of 6 months. All patients underwent abdominal fat packing in the sphenoid sinus to minimize the risk of postoperative CSF leakage.

Removal of pituitary adenomas was followed by sphenoid sinus fat packing using the following previously-described procedures: ${ }^{1415)}$ 1) autologous fat was obtained from the abdomen; 2) the sphenoid sinus mucosa was complete- ly removed under microscopic or endoscopic control; 3) the sphenoid sinus was packed with the harvested abdominal fat; and 4) the elevated septal mucosa was repositioned. All surgical procedures were performed at our institution by one neurosurgeon (W.K.) and one otolaryngologist (P. D-. J.).

\section{Postoperative MRI}

Within $48 \mathrm{~h}$ of surgery, all patients underwent postoperative MRI scans of the sella using either a 1.5-Tesla system (Signa; General Electric, Minneapolis, Minnesota, USA) or a 3.0-Tesla system (Achieva; Philips Healthcare, Best, the Netherlands). Follow-up MRI scans were performed 6 months and 1 year after surgery and every other year thereafter. We measured the maximal orthogonal diameter of the transplanted fat in each of the following planes: axial (Daxi), sagittal (Dsag), and coronal (Dcor). The volume of transplanted fat in the sphenoid sinus was calculated using the following equation: $V=$ Daxi $\times$ Dsag $\times$ Dcor $\times \pi / 6$. Finally, we estimated the fat distribution percentage relative to the initial volume obtained from the postoperative scans. Five fat clearance scores were allocated to each image using a modified system developed in a previous study, with ranges of $0-20 \%, 21-40 \%, 41-60 \%, 61-80 \%$, and $81-100 \%$ fat tissue. ${ }^{16)}$ Furthermore, the extent of tumor resection, recurrent or remnant tumor regrowth, and the development of inflammatory complications were also determined, based on the postoperative MRI findings. The sphenoid sinus mucocele was excluded due to the absence of growth over time and by additional application of the fat-suppression sequence. ${ }^{17) 18)}$ All assessments of MRI images were performed by two independent investigators in a blinded manner (H.S.L. and C.S.L.).

\section{Outcome measures and statistical methods}

The following details were collected from the patients' medical records: sex, age, major presenting symptoms, comorbidities, subsite of tumor origin, length of hospital stay, and postoperative complications. The mean follow-up period was calculated from the day after completion of the primary treatment. "No residual tumor" was defined as the absence of clinical, radiological, or pathological evidence of persistence or recurrence within the original tumor bed following surgery. Fat clearance analyses included data for patients who died from causes unrelated to the tumor.

All continuous data are presented as the mean \pm standard deviation. The Student's t test was used to compare contin- 
uous variables. Pearson's $\chi^{2}$ test or Fischer's exact tests were used to compare categorical variables. Data analyses for estimating fat clearance were performed by Kaplan-Meier time-to-event analysis and Cox regression analysis. The inter-observer agreement for each imaging feature was calculated using the intra-class correlation coefficient and Cronbach's $\alpha$ coefficient. All tests used a $p$ value of 0.05 as the significance threshold, and all statistical analyses were conducted using PASW Statistics for Windows, Version 18.0 (SPSS Inc., Chicago, IL, USA).

\section{RESULTS}

\section{Patient characteristics}

The clinical and demographic data of the patients are summarized in Table 1. Among the 41 patients enrolled, 18 were men and 23 were women (age range, 23-77 years; mean, $57.2 \pm 13.4$ years). The study sample included two

Table 1. Summary of patients' clinical information

\begin{tabular}{lc}
\hline Mean age at diagnosis (range), years & $57.2 \pm 13.4(23-77)$ \\
Sex, $\mathrm{n}(\%)$ & $18(43.9)$ \\
Male & $23(56.1)$ \\
Female & $14.2 \pm 8.6(8-51)$ \\
Length of hospital stay (range), days & $2(4.9)$ \\
Revision cases, $\mathrm{n}(\%)$ & $17(41.5)$ \\
Residual mass, $\mathrm{n}(\%)$ & $3(7.3)$ \\
Postoperative complications, $\mathrm{n}(\%)$ & $2(4.9)$ \\
CSF rhinorrhea & $1(2.4)$ \\
Septal perforation & $51.9 \pm 30.0(9-137)$ \\
Sphenoid sinusitis & $33.4 \pm 21.9(6-94)$ \\
Mean follow-up duration (range), \\
months \\
Mean time between surgery and \\
the last MRI (range), months \\
$\begin{array}{l}\text { Mean number of postoperative MRI } \\
\text { scans (range) }\end{array}$ \\
\hline MRI: magnetic resonance imaging, CSF: cerebrospinal fluid
\end{tabular}

revision cases (4.9\%) and 17 patients $(41.5 \%)$ with residual tumors. CSF rhinorrhea represented the leading postoperative complication in three patients $(7.3 \%)$, followed by two septal perforations and one sphenoid fungal sinusitis. No patients presented with sphenoid sinus mucoceles; optic chiasmal compression by fat packing; or donor site complications such as wound dehiscence, hematoma, or infection. For the entire study population, the mean follow-up period was $51.9 \pm 30.0$ months (range, $9-137$ months). The mean interval between surgery and the final MRI scan of each patient was $33.4 \pm 21.9$ months (range, 6-94 months) and the mean number of postoperative MRI scans for each patient was $4.4 \pm 1.0$.

\section{Clearance of fat transplanted into the sphenoid sinus using postoperative MRI assessments}

In total, 139 postoperative MRI scans from 41 patients were acquired and evaluated. The inter-observer agreement for the fat clearance scores was within a statistically excellent range, and the intra-class coefficient between the two investigators was $0.89(0.82-0.94, \mathrm{p}<0.001)$. The percentage categories of remaining fat tissue for each time interval are provided in Table 2; the overall tendency was for the fat content to be reduced over time. The amount of fat tissue visualized on the final MRI scan was $<20 \%$ in the majority of cases $(34 / 41,82.9 \%)$ and $>60 \%$ in only $4.9 \%$ $(2 / 41)$ of cases. The median half-life of transplanted fatty tissue was 18.0 months (Fig. 1A). The fat-transplanted sphenoid sinus was eventually filled with air, and neither mucocele formation nor necrotic oil cysts occurred in any of the participants.

The influences of clinical and demographic factors on fat clearance rates were assessed in a subgroup analysis. Only the presence of residual tumor exerted a significant effect on the fat clearance rate (Fig. 1B). The calculated median half-life of viable fat tissue was significantly longer in pa-

Table 2. Time interval between surgery, final magnetic resonance imaging scan, and volume of visualized fat tissue

\begin{tabular}{ccccccc}
\hline \multirow{2}{*}{ Time between surgery and final MRI scan (months) } & \multicolumn{5}{c}{ Percentage of remaining fat tissue (\%) } \\
\cline { 2 - 6 } & $0-20$ & $21-40$ & $41-60$ & $61-80$ & $81-100$ & Total \\
\hline $6-12$ & 2 & 2 & 1 & 1 & 14 \\
$13-24$ & 12 & 1 & 1 & 1 & 3 \\
$25-48$ & 14 & & & 3 \\
$49-72$ & 3 & & 2 & 41 \\
$73-96$ & 3 & & 3 & 2 & 3 \\
Total & 34 & 3 & & \\
\hline
\end{tabular}

MRI: magnetic resonance imaging 
tients with residual tumors than in those without remnant tumors (median half-lives of 21.8 and 17.2 months, respectively, $\mathrm{p}=0.013$ ). Representative postoperative MRI features of fat grafts in the sphenoid sinus with and without residual tumors are provided in Fig. 2. Patient age distribution, sex, and the extent of the tumor (that was proportional to the

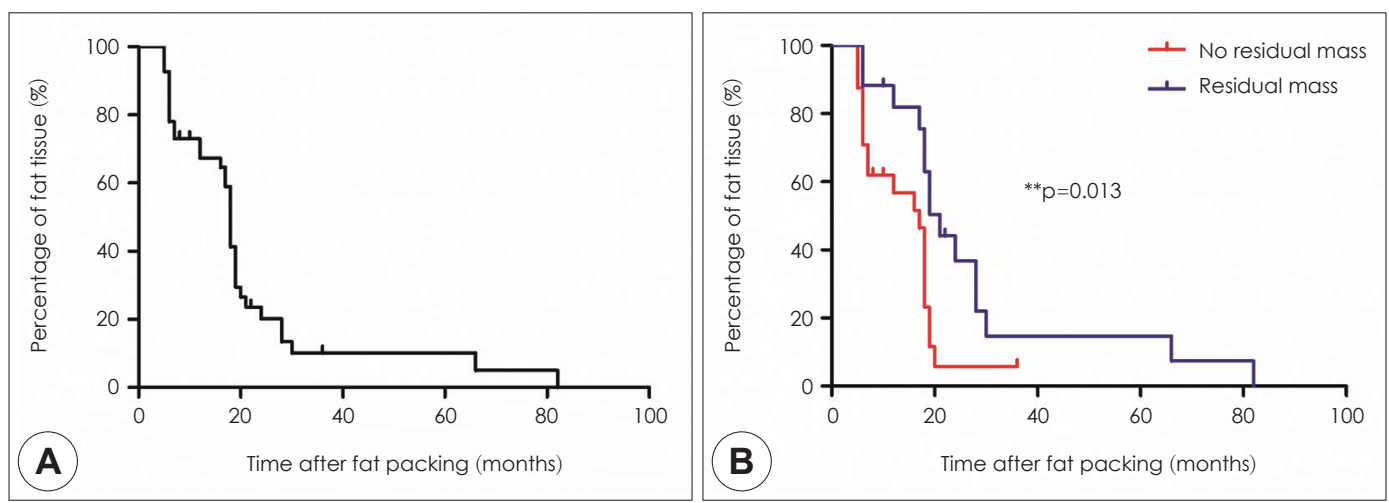

Fig. 1. The clearance of fat transplanted into the sphenoid sinus using postoperative magnetic resonance imaging (MRI) assessments. A: The overall tendency was for the fat tissue volume to be reduced over time, as measured by postoperative MRl. B: Comparison of the percentage of remaining fat tissue between participants with (blue line) and without (red line) residual tumors. Each time series was assessed by Kaplan-Meier time-to-event analysis. **: $p<0.05$.
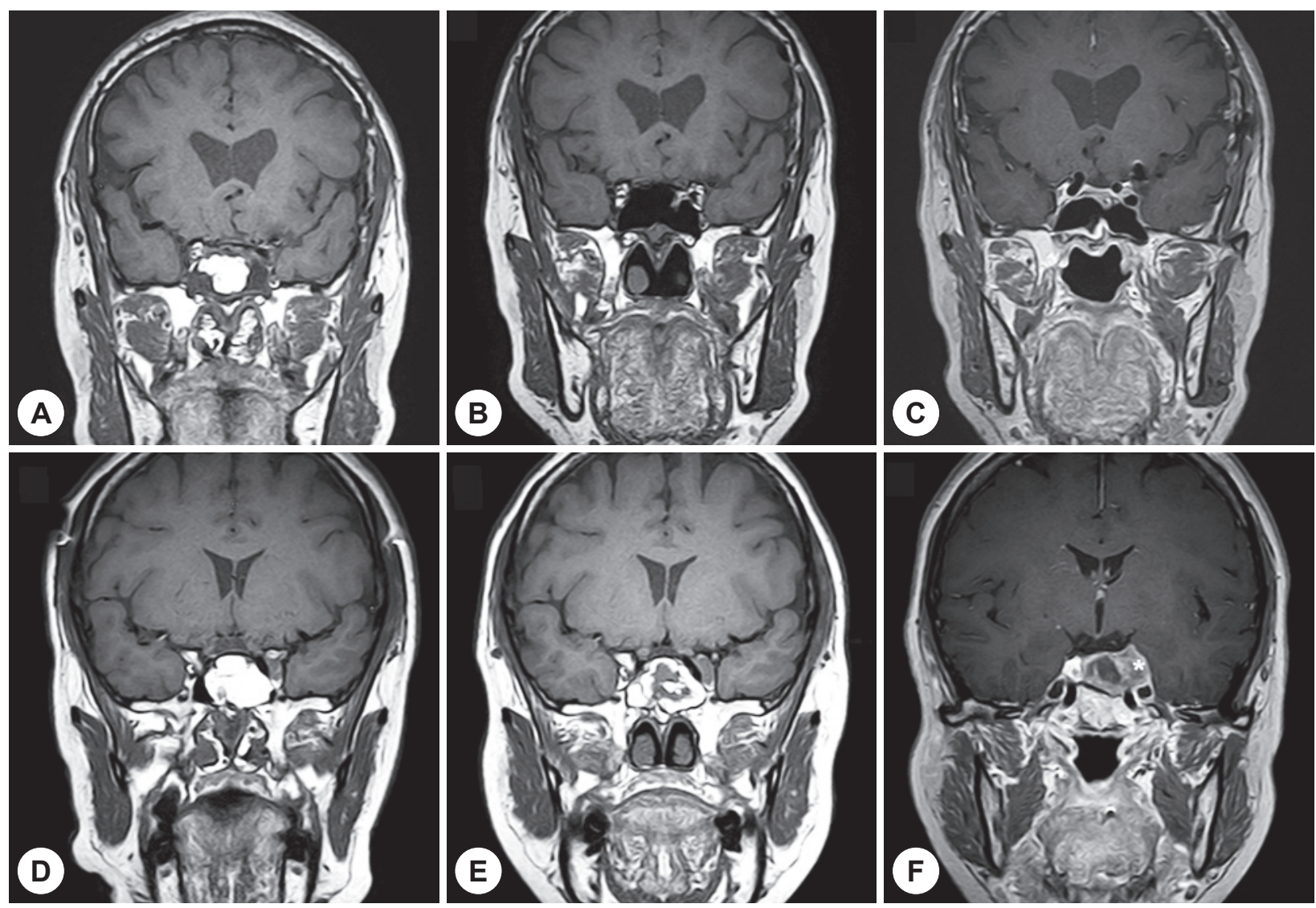

Fig. 2. Representative postoperative magnetic resonance images of fat grafts in the sphenoid sinus in the presence and absence of remnant tumors. A: The immediate postoperative coronal Tl-weighted magnetic resonance imaging (MRI) scan indicates surviving fat tissues as white areas in the sphenoid sinus. B: While the viable fat tissues have nearly been reabsorbed 17 months after surgery (coronal T1-weighted). C: Neither demonstrable abnormal space-occupying lesions nor signal changes in the operative field can be observed (coronal Tl-gadolinium-enhanced image). For cases in which the pituitary fumor was only partially removed. D: The immediate postoperative coronal Tl-weighted MRI scan reveals a hyper-intense fat signal protruding from inside the sphenoid sinus and the anterior aspect of the sella turcica. E: Fat contents are visible at 18 months postoperatively (coronal T1-weighted image). F: The postoperative 18-month coronal Tl-gadolinium-enhanced MRI scan indicates a heterogeneous enhancing solid mass with extension of the left parasellar extension (asterisk) i.e., a suspected residual adenoma. 
transplant volume) did not significantly affect the visibility period of the implanted fat.

\section{DISCUSSION}

Over the past 20 years, the use of autologous fat grafting has become an attractive technique in both aesthetic and reconstructive surgery because it is easier to perform and the risk of adverse tissue reactions is low. However, one of the major disadvantages of this technique is the unpredictable fat resorption rate. Since there is no standardized fat graft technique encompassing harvesting, processing, storage, and managing the recipient site, a number of clinical and experimental studies of fat grafting have reported differences in graft survival. ${ }^{19)}$ Given the esthetic purpose of fat grafting, it is not possible to perform serial MRI scans, which is presently considered to be the best imaging method to assess fat distribution in terms of objective clinical efficacy. Hence, there is a lack of in-depth knowledge regarding the factors influencing fat grafting outcomes. ${ }^{1019)}$ Although the fat grafting procedure employed in our study was not aimed to restore soft tissue loss as in cosmetic surgery, we aimed to evaluate the fat graft survival rate in the sphenoid sinus using MRI follow-up.

The obliteration of the frontal sinus with fat following osteoplastic flap surgery is highly effective for the most severe and recalcitrant cases of frontal sinusitis. ${ }^{20)}$ Weber et al. ${ }^{16)}$ reported the long-term results of osteoplastic frontal sinus surgery with fat obliteration, using 86 postoperative MRI scans. The fat tissue volume decreased significantly with time, with a median half-life of 15.4 months, which concurred with our observations. Constantinidis et al. ${ }^{21)}$ used an animal model with obliteration of the maxillary sinus with fat by means of histopathologic analysis and MRI. In rabbits, the study group received abdominal fat transplanted to the maxillary sinus following osteoplastic flap procedures. The volume of fat tissue decreased by $1-30 \% 6$ months after the transplantation relative to the post-operative volumes, and microscopic images predominantly indicated fat neogenesis and newly-created lamellar bone in the bony cavity. This discrepancy between studies may have arisen because the fate of transplants depends on the surgical technique as well as transplant handling. In our study, removal of the sinus mucosa or drilling of the internal bony layer before transplantation might have inhibited the subsequent revascularization capability, thereby affecting the viability of the fat cells. ${ }^{22)}$

Interestingly, in the patients with residual tumors in our study, follow-up MRI scans revealed significantly delayed fat resorption. Even at the long-term follow-up intervals, considerable volumes of non-resorbed fat remained within the sphenoid sinus, as indicated by the isointense enhancement of tumors. There is increasing evidence that tumor cell and lipid biology interactions influence numerous cellular processes, including cell growth, proliferation, and differentiation. ${ }^{2324)}$ During the adipogenic cascade, the signal transduction pathway is regulated by a large number of hormones. ${ }^{12)}$ Fat cell survival may have been enhanced by inhibition of apoptosis and provision of paracrine stimuli by the nearby residual tumors. Further investigations using animal models are required to investigate the influence of tumors on the metabolic processes of fat tissue.

Our study of 139 postoperative MRI scans of pituitary adenomas from a single institution constitutes a database with uniform diagnostic and therapeutic management policies applied by the same body of surgeons. This is the strength of our study, although as a retrospective analysis it is susceptible to the shortcomings in data collection that are possible in such study designs. Further randomized controlled trials are needed to clarify the effects of other factors on the vitality and persistence of transplanted fat, including the anatomical site and different harvesting methods.

\section{CONCLUSION}

Together with observations reported in the literature, our results supply further evidence that the volume of surviving sphenoidal fat transplants decreases over time. The fattransplanted sphenoid sinus was ultimately almost filled with air, with no formation of mucoceles. However, cases with residual pituitary tumors exhibited slower declines in fat volume. Our preliminary data strengthen the clinical information regarding fat graft survival and demonstrate grafting outcome predictability.

\section{Author Contributions}

Conceptualization: Dong-Joon Park, Kum Whang. Data curation: Hee Sung Chae. Formal analysis: Hyun Su Lee, Chung Seok Lee. Methodology: Chi Sang Hwang. Visualization: Hee Sung Chae, Chi Sang Hwang. Writing - original draft: Chi Sang Hwang. Writing - review \& editing: Young Joon Seo, Dong-Joon Park. 


\section{REFERENCES}

1) Ezzat S, Asa SL, Couldwell WT, Barr CE, Dodge WE, Vance ML, et al. The prevalence of pituitary adenomas: a systematic review. Cancer 2004;101:613-9.

2) Molitch ME. Nonfunctioning pituitary tumors. Handb Clin Neurol 2014;124:167-84.

3) Sonnenburg RE, White D, Ewend MG, Senior B. The learning curve in minimally invasive pituitary surgery. Am J Rhinol 2004;18:25963.

4) Arita K, Kurisu K, Tominaga A, Ikawa F, Iida K, Hama S, et al. Sizeadjustable titanium plate for reconstruction of the sella turcica. Technical note. J Neurosurg 1999;91:1055-7.

5) Cappabianca P, Cavallo LM, Mariniello G, de Divitiis O, Romero $\mathrm{AD}$, de Divitiis E. Easy sellar reconstruction in endoscopic endonasal transsphenoidal surgery with polyester-silicone dural substitute and fibrin glue: technical note. Neurosurgery 2001;49:473-5; discussion 5-6.

6) Couldwell WT, Kan P, Weiss MH. Simple closure following transsphenoidal surgery. Technical note. Neurosurg Focus 2006;20:E11.

7) Chen HC, Lee ST. Need for intrasellar packing in sellar reconstruction of transsphenoidal surgery: less is more? J Clin Neurosci 2006; 13:423-7.

8) Seda L, Camara RB, Cukiert A, Burattini JA, Mariani PP. Sellar floor reconstruction after transsphenoidal surgery using fibrin glue without grafting or implants: technical note. Surg Neurol 2006;66:46-9; discussion 9.

9) Farrell CJ, Nyquist GG, Farag AA, Rosen MR, Evans JJ. Principles of Pituitary Surgery. Otolaryngol Clin North Am 2016;49:95-106.

10) Sinno S, Wilson S, Brownstone N, Levine SM. Current Thoughts on Fat Grafting: Using the Evidence to Determine Fact or Fiction. Plast Reconstr Surg 2016;137:818-24.

11) Sezgin B, Ozmen S, Bulam H, Omeroglu S, Yuksel S, Cayci B, et al. Improving fat graft survival through preconditioning of the recipient site with microneedling. J Plast Reconstr Aesthet Surg 2014; 67:712-20.

12) Doornaert M, Colle J, De Maere E, Declercq H, Blondeel P. Autologous fat grafting: Latest insights. Ann Med Surg (Lond) 2019;37:
47-53

13) Carpaneda CA, Ribeiro MT. Percentage of graft viability versus injected volume in adipose autotransplants. Aesthetic Plast Surg 1994; 18:17-9.

14) Fatemi N, Dusick JR, de Paiva Neto MA, Kelly DF. The endonasal microscopic approach for pituitary adenomas and other parasellar tumors: a 10-year experience. Neurosurgery 2008;63:244-56; discussion 56.

15) Roca E, Penn DL, Safain MG, Burke WT, Castlen JP, Laws ER Jr. Abdominal Fat Graft for Sellar Reconstruction: Retrospective Outcomes Review and Technical Note. Oper Neurosurg (Hagerstown); 2018.

16) Weber R, Draf W, Keerl R, Kahle G, Schinzel S, Thomann S, et al. Osteoplastic frontal sinus surgery with fat obliteration: technique and long-term results using magnetic resonance imaging in 82 operations. Laryngoscope 2000;110:1037-44.

17) Tien RD. Fat-suppression MR imaging in neuroradiology: techniques and clinical application. AJR Am J Roentgenol 1992;158:369-79.

18) Hansen FS, van der Poel NA, Freling NJM, Fokkens WJ. Mucocele formation after frontal sinus obliteration. Rhinology;2018.

19) Gir P, Brown SA, Oni G, Kashefi N, Mojallal A, Rohrich RJ. Fat grafting: evidence-based review on autologous fat harvesting, processing, reinjection, and storage. Plast Reconstr Surg 2012;130:24958.

20) Eloy JA, Marchiano E, Vazquez A. Extended Endoscopic and Open Sinus Surgery for Refractory Chronic Rhinosinusitis. Otolaryngol Clin North Am 2017;50:165-82.

21) Constantinidis J, Bohr C, Greess H, Aigner T, Zenk J, Prokopakis E, et al. Fat obliteration in paranasal sinuses: a comparative magnetic resonance imaging and histopathologic study. Laryngoscope 2005;115:717-23.

22) Shiffman MA, Mirrafati S. Fat transfer techniques: the effect of harvest and transfer methods on adipocyte viability and review of the literature. Dermatol Surg 2001;27:819-26.

23) Santos CR, Schulze A. Lipid metabolism in cancer. FEBS J 2012; 279:2610-23.

24) Porstmann T, Santos CR, Griffiths B, Cully M, Wu M, Leevers S, et al. SREBP activity is regulated by mTORC1 and contributes to Aktdependent cell growth. Cell Metab 2008;8:224-36. 